\title{
Manganese-enhanced MRI depicts a reduction in brain response to nociception by mTOR inhibition in chronic pain rats
}

Myeounghoon Cha ( $D$ mhcha@yuhs.ac)

Yonsei University https://orcid.org/0000-0002-7993-672X

Songyeon Choi

Yonsei University College of Medicine

Kyeongmin Kim

Yonsei University College of Medicine

Bae Hwan Lee

Yonsei University College of Medicine

\section{Research}

Keywords: MEMRI, mTOR, chronic pain, Torin1, XL388

Posted Date: June 12th, 2020

DOI: https://doi.org/10.21203/rs.3.rs-33778/v1

License: (c) (i) This work is licensed under a Creative Commons Attribution 4.0 International License.

Read Full License 


\section{Abstract}

Neuropathic pain induced by a nerve injury could lead to chronic pain. Recent studies have reported hyperactive neural activities in the nociceptive-related area of the brain as a result of chronic pain. Although cerebral activities associated with hyperalgesia and allodynia in the chronic pain model were difficult to represent with functional imaging techniques, advances in manganese (Mn)-enhanced magnetic resonance imaging (MEMRI) could facilitate the visualization of the activation of pain-specific neural responses in the cerebral cortex. In order to investigate the alleviation of pain nociception by mammalian target of rapamycin (mTOR) modulation, we observed the cerebrocortical excitability changes and compared the regional Mn 2+ enhancement after mTOR inhibitions. At day 7 after nerve injury, drugs were applied into the intracortical area, and drug (Vehicle, Torin1 and XL388) effects were compared within groups using MEMRI. In the results, signal intensities of the insular cortex (IC), primary somatosensory cortex of the hind limb region (S1HL), motor cortex 1/2 (M1/2), and anterior cingulate cortex (ACC) regions were significantly reduced after application of mTOR inhibitors (Torin1 and XL388). Furthermore, the rostral-caudal analysis of the IC indicated that the rostral region of the IC was more associated with pain perception than caudal region. Our data suggest that MEMRI could present the painrelated signal changes in the brain, and mTOR inhibition is closely correlated with pain modulation in chronic pain rats.

\section{Introduction}

Neuropathic pain arises from an initial injury, such as neuropathy caused by a lesion of, or damage to, the somatosensory nervous system, which can also lead to chronic pain [1]. Chronic pain may depend on plastic reorganization of the central nervous system (CNS). Investigation of changes in the cerebral neuronal activity is a typical challenge for functional imaging, and uncovering the brain abnormalities underlying the chronic pain sensation could be considered as a first step in clinical approach [2]. Magnetic resonance imaging (MRI) techniques are essential tools for the study of cerebral activities. Current manganese-enhanced MRI (MEMRI) tools have provided a visible method for demonstrating the cortical response after evoked and/or spontaneous pain [3,4]. The chemical properties of $\mathrm{Mn}^{2+}$ resemble those of $\mathrm{Ca}^{2+}$, and $\mathrm{Mn}^{2+}$ acts as a paramagnetic neuronal tract tracer as its transport uses voltage-gated $\mathrm{Ca}^{2+}$ channels throughout the nervous system [5-7]. Experimental hyperalgesia produces the up-regulated neuronal activation of brain response within pain-processing regions, including the anterior cingulate cortex (ACC), insular cortex (IC), as well as primary (SI) and secondary somatosensory cortex (SII) $[2,8,9]$. Since MEMRI studies were used to demonstrate the identifying pain pathways in the brain, various pain imaging studies have been performed in the spinal cord and brain [6, 7]. Nerve injury [10] and thermal [11] stimulus-induced abnormal brain activities have been shown using MEMRI. In addition, our previous formalin-induced pain study also showed that MEMRI could be a good indicator of pain-induced changes in the CNS [7].

Mammalian target of rapamycin (mTOR), a serine-threonine protein kinase, is known to regulate mRNA translation in the nervous system, and it plays important roles in cell proliferation and differentiation [12]. 
Although the roles of mTOR pathway in cancer research have been extensively studied, there has been no in-depth study on the role of mTOR signaling in pain research. Recent studies have reported that neuropathic pain is related to structural changes in the CNS after nerve injury, known as mTOR-mediated neuronal plasticity [13-16]. The activation of MTOR regulates protein synthesis and influences a wide range of physiological and pathological states by phosphorylating downstream effectors $[12,17,18]$. In this study, the mTOR selective inhibitors known as Torin1 $[19,20]$ and XL388 [21] were used to modulate the mTOR regulator. Each agent has highly potent and ATP-competitive mTOR inhibitory effects.

Here, we investigated the changes of cerebrocortical excitability in nerve injury-induced chronic pain using MEMRI, and compared the noxious stimulation-dependent regional signal changes after mTOR inhibitors applications within groups using $\mathrm{Mn}^{2+}$ enhancement. In particular, we analyzed the different distributions of $\mathrm{Mn}^{2+}$ enhancement in the rostral and caudal parts of the region of interest (ROI), which has been implicated to be associated with different roles in pain perception. These findings may contribute to the knowledge of hyperalgesia in the brain by nerve injury-induced chronic pain, and may suggest potential therapeutic target using mTOR inhibition in chronic pain.

\section{Materials And Methods}

\subsection{Animal preparation}

Male Sprague-Dawley rats ( $n=24,250-300 \mathrm{~g}$, Koatech, Pyeongtaek, Korea) were used in this experiment. All animal experiments were approved by the Institutional Animal Care and Use Committee (IACUC) of the Yonsei University Health System (protocol number 2016-0061). All experiments were performed in accordance with the IACUC guidelines and regulations. Animals were housed in plastic cages with soft bedding on a 12-hour light/dark cycle (light cycle: 08:00 20:00), at a constant temperature $\left(22 \pm 2^{\circ} \mathrm{C}\right.$ ) and humidity ( $50 \pm 10 \%$ ). Experimental procedure according to the time sequence is summarized in Fig. 1 . Rats were anesthetized with an intraperitoneal (i.p.) injection of sodium pentobarbital $(50 \mathrm{mg} / \mathrm{kg}$ ). Deep anesthesia was verified by loss of nociception in response to a tail pinch stimulation. The surgical procedure for nerve injury was performed following the methods described in our previous report [22, 23]. The left sciatic nerve was exposed, and three major divisions were clearly separated. Then, the tibial and sural nerves were tightly ligated and transected. Complete hemostasis was confirmed, and the wound was closed with muscle and skin sutures.

Changes in the mechanical paw withdrawal threshold were measured before nerve injury (pre) and at 1, 4, and 7 days after nerve injury. Rats were habituated for $10 \mathrm{~min}$ to the testing cages, which consisted of metal mesh floors under plastic domes. Mechanical allodynia was measured by assessing thresholds for hind paw withdrawal upon stimulation with an electrical von Frey (Ugo Basile, Varese, Italy). Mechanical forces were recorded with each withdrawal. Responses were measured seven times, and the means were calculated after the maximum and minimum values were excluded (supplementary fig.1).

\subsection{Agent administration}


On postoperative day 7 , all rats were anesthetized with urethane $(0.5 \mathrm{~g} / \mathrm{kg}$, i.p.) and a-chloralose (25 $\mathrm{mg} / \mathrm{kg})$. In addition, atropine $(20 \mu \mathrm{g} / \mathrm{kg}$, i.p.) was injected to avoid excessive mucus secretion in the trachea. The depth of anesthesia was determined by a lack of flexor muscle response to hind-paw pinching. Then, the rats were mounted on the surgical stage. Skin incision was performed on the right ventral aspect of the neck, and the common carotid artery and external carotid artery (ECA) were gently exposed. Polyethylene tubing (PE-10) was cannulated into the ECA.

Following catheter insertion, a $20 \%$ D-mannitol solution $\left(35 \pm 2^{\circ} \mathrm{C}, 5 \mathrm{ml} / \mathrm{kg}\right.$, Dai Han Pharm, Seoul, Korea) was infused to open the blood-brain barrier. To investigate pain-dependent signal changes in the whole brain following mTOR inhibition, nerve-injured rats were divided into three groups (Control, $n=8$; Torin1, $\mathrm{n}=8$ and $\mathrm{XL388}, \mathrm{n}=8)$. Then, the vehicle (0.06\% DMSO in saline, $1 \mathrm{ml})$, Torin1 (400 $\mathrm{nM}$ in vehicle, $1 \mathrm{ml}$ ), and XL388 (500 nM in vehicle, $1 \mathrm{ml}$ ) were infused in each group of rats. Following the application, $20 \mathrm{mM}$ of manganese chloride $\left(\mathrm{MnCl}_{2}-4 \mathrm{H}_{2} \mathrm{O}\right.$, Sigma, St. Louis, $\left.\mathrm{MO}, \mathrm{USA}\right)$ was injected for MR imaging. All infusions were injected via the ECA (150 $\mu \mathrm{l} / \mathrm{min})$ using a syringe pump (22 Infusion Syringe Pump, Harvard Apparatus, Holliston, MA, USA). For noxious stimulation, electric stimulations ( $3 \mathrm{~mA}, 2 \mathrm{~Hz}, 1 \mathrm{~ms}$ width; A385, WPI, Sarasota, FL, USA) were applied to the left hind paw during manganese chloride injection.

\subsection{MR measurements}

MR imaging was performed using a 9.4-T horizontal Biospec small bore scanner (Bruker BioSpin, Ettlingen, Germany) with an $86-\mathrm{mm}$ volume coil to transmit the radio frequency (RF) and a four-channel array coil to receive the signal. After tuning and shimming, T2 multi-slice spin echo sequence images were acquired for positioning. T1W images were taken at the same positions. High-resolution anatomical images were obtained with a rapid acquisition with relaxation enhancement (RARE) protocol using the following parameters: Effective TE $=28 \mathrm{~ms}, \mathrm{TR}=4750 \mathrm{~ms}, \mathrm{FOV}=32 \times 32 \mathrm{~mm}$, matrix $=192 \times 192$, and RARE factor $=8$. Fifteen slices of T1W images were acquired using the RARE protocol with shortened TR. The imaging parameters were RARE factor $=2, T E / T R=8 / 412 \mathrm{~ms}, \mathrm{FOV}=32 \times 32 \mathrm{~mm}$, matrix $=192 \times 192$, and slice thickness $=1 \mathrm{~mm}$. Respiration rates of animals were monitored during the experiments.

Functional analysis was performed using ParaVision (Version 5.0, Bruker BioSpin). The whole brain was outlined on axial slices, and the mean signal was calculated. To quantify data from the series of T1W images and measure the $\mathrm{Mn}^{2+}$ enhancement in separated brain regions, ROls were set to the entire brain regions that were covered in coronal images. ROIs, the insular cortex (IC), primary somatosensory cortex of the hind limb (S1HL), motor cortex 1/2 (M1/2), anterior cingulate cortex (ACC), and visual cortex 1/2 (V1/2) were manually drawn on each separate image. Signal magnitudes from each brain region were normalized to signal intensity in the temporalis muscle. Image analysis was performed using ParaVision (Bruker Biospin), MRVision (MRVision Co., Winchester, MA, US), and ImageJ software (National Institutes of Health, Bethesda, MD, US). Data are presented as mean \pm standard error of the mean (SEM). After MRI scans were taken, the rats were humanely killed with an overdose of urethane $(1.5 \mathrm{mg} / \mathrm{kg})$.

\subsection{Statistical analysis}


To quantify the data from T1W images and the measurement of $\mathrm{Mn}^{2+}$ enhancement in the brain, each $\mathrm{ROI}$ was set to the brain atlas covered in coronal images. Relative signal intensities were calculated and compared within groups. Data are presented as mean \pm SEM. One-way analysis of variance (ANOVA) followed by Dunnett's post-hoc pairwise comparisons were used to identify the mean difference of the ROI contrast. All statistical analyses were performed with Prism 5 software (Version 8.03; GraphPad software Inc, San Diego, CA, US). P $<0.05$ indicated a statistically significant difference.

\section{Results}

\subsection{Analysis of Mn-enhanced MR signals}

All of the acquired brain images exhibited regional heterogeneity in signal distribution typical of $\mathrm{MnCl}_{2}$ administration. The diffusion of $\mathrm{Mn}^{2+}$ was found in the medial ventral region of the brain, hypothalamus, and specific pain-related structures. High-signal intensities were observed in the IC, S1HL, M1/2, and ACC (Fig. 2). V1/2 areas were also collected for the stimuli-independent ROI. These regions were selected for further ROI analysis. Fig. 2 illustrates ROIs from which average signal intensity was extracted (several ROIs extend over several slices). In Fig. 3, the slice-dependent Mn-enhanced images of each group were compared (Fig. 3). During the comparison, high Mn-enhanced signals were observed in control (vehicleinfused) rats in all of the acquired slices. However, Torin1- and XL388-injected rats did not present painrelated Mn-enhanced signals in the IC, S1HL, M1/2, and ACC regions.

Fig. 4A shows the representative coronal (Fig. 4A upper left), sagittal (Fig. 4A right), and transverse (Fig. 4A left bottom) images of control rats (Fig. 4A). Across the brain regions, Mn-enhanced signals were significantly enhanced in the right hemisphere of contralateral for nerve injury and peripheral stimulation. In control group, the IC, S1HL, M1/2, and ACC revealed higher mean signal intensities compared to other groups. This indicated that large quantities of $\mathrm{Mn}^{2+}$ ions were transported and accumulated in each region. Regional signal intensity comparisons are presented in Figures 4B to 4F (Fig. 4B: IC, 4C: S1HL, 4D: M1/2, 4E: ACC, and 4F: V1/2). In contrast, signal intensity of Torin1- and XL388-infused groups showed less enhanced signal intensities in the IC, $\mathrm{S} 1 \mathrm{HL}, \mathrm{M} 1 / 2$, and $\mathrm{ACC}$. $\mathrm{P}<0.05$ indicated significant difference between drugs versus control group (ANOVA followed by Dunnett's post-hoc test). Furthermore, the mean signal intensities in the IC, S1 HL, M1/2, and ACC did not differ between Torin1 and XL388 groups. The mean signal intensities of the $\mathrm{V} 1 / 2$ remained less-enhanced than those in other ROls and were similar between the three groups, with no statistically significant differences (Fig. 4F). The signal intensities of the ipsilateral primary somatosensory cortex of jaws (S1 J) were higher in all groups of animals. However, it was not related to the pain caused by the given hind paw stimulus, and was excluded from data analysis. Each value of the signal intensities is presented in Table 1.

\subsection{Comparison of Mn-enhanced signal expressions}

To elucidate the differences between groups, we analyzed the integrated signal values of ROIs, and the IC and $\mathrm{M} 1 / 2$ regions were divided into rostral-caudal parts and analyzed. Comparison of regional signal 
intensities between groups is presented in Fig. 5. Stimulus-dependent Mn-enhanced signal intensities were significantly reduced after Torin 1 and XL388 infusion, and Mn-enhanced signals after hind paw stimulation in the IC appeared higher in the rostral planes (Fig. 5A, bregma 2 and $1 \mathrm{~mm}$ ) than in the caudal planes (Fig. 5B, bregma -1 and $-2 \mathrm{~mm}$ ) of the IC. In the S1 HL, which directly received painful nociception signals after stimuli, we could observe an increased signal value in the whole planes of control groups. However, significant reductions were observed in the Torin1- or XL388-infused rats (Fig. 5C). In the M1/2 rostral-caudal analysis comparison, Torin1- and XL388-infused rats showed regional specific Mn-enhancement in the M1/2 area compared to control rats (Fig. 5D and 5E,). At the caudal part of the $M 1 / 2$ (Fig. $5 E$, bregma $-1,-2$, and $-3 \mathrm{~mm}$ ) planes, there was no significant difference between groups. However, for the rostral part of M1/2 (Fig. 5D, bregma 2 and $1 \mathrm{~mm}$ ) planes, data from Torin 1 - or XL388-injected groups showed a significantly decreased value (Fig. 5D, ANOVA followed by Dunnett's post-hoc test). In addition, in the comparison of signal intensities in the ACC, Torin1- or XL388-treated groups showed significantly reduced signal enhancements in the rostral part (Fig. 4F, bregma 2 and 1 $\mathrm{mm}$ ). However, the V1/2 signal intensities in pain-independent brain regions showed no difference in all groups.

\subsection{Stimulus-induced signal intensities in ROls}

In order to quantify the stimulus-dependent signal intensity changes, signal intensities in ROls were compared to the signal intensities of the V1/2 regions of the same animals (Fig. 6). In signal intensity comparisons between the rostral area of the IC and the caudal area of the IC, the rostral area of the IC showed more enhanced signal activities when painful stimuli were given to the hind paw compared to the caudal area of the IC. This signal enhancement was more explicit when compared to the V1/2 (Fig. 6A; rostral-IC $2.04 \pm 0.28$, caudal-IC $1.42 \pm 0.19$, and V1/2 $1.47 \pm 0.11$ ). In addition, the S1HL directly reflected hind paw stimulation, and signal difference with $\mathrm{V} 1 / 2$ was greater compared to those in other regions (Fig. 6B; S1HL $2.59 \pm 0.25$, and V1/2 $1.47 \pm 0.11$ ). In comparison of the mean signal enhancement in the $\mathrm{M} 1 / 2$ region, the rostral part of the M1/2 region showed more enhanced activities compared to the caudal part of M1/2 region (Rostral-M1/2 $2.20 \pm 0.22$ vs. Caudal-M1/2 $1.48 \pm 0.14$ ). However, it was not significant in comparison with the $\mathrm{V} 1 / 2$ region. In addition, the ACC also showed no significant difference compared to the $\mathrm{V} 1 / 2$ signal intensities (Fig. 6D ACC $2.07 \pm 0.17$ ).

\section{Discussion}

The purpose of this study was to evaluate the usefulness of MEMRI in chronic pain research regarding the whole brain analysis, as well as to measure the changes in brain activities after drug applications under conditions of chronic pain. In this study, we demonstrated the cortico-cortical neural excitation of several ROls under chronic neuropathy using MEMRI and the changed Mn-enhanced signals of the painrelated cortex area after direct infusion of mTOR inhibitors in the brain. Until now, the effects of mTOR inhibitor using animal MRI have not been studied. Several studies have used the MEMRI method to assess pain in the central nervous system or to observe changes in pain through analgesic use $[4,10,24]$. However, the MEMRI method has several difficulties in observing the effects of pain reduction or changes 
in neuronal activities. In order to abbreviate these problems, we tried to observe dynamic changes in the brain using ECA injection with $\mathrm{MnCl}_{2}$.

\subsection{The meaning of $\mathrm{Mn}^{2+}$ enhancement in ROIs}

MEMRI has advantages in the fast influx and enhancements of $\mathrm{Mn}^{2+}$ in the tissues. [24] In addition, the cumulative $\mathrm{Mn}^{2+}$ pattern is retained for several days, and it also provides a good opportunity to observe activated brain changes by stimulation when viewed through T1-weighted MRI [6]. Quantitative evaluation of contrast enhancement showed that $\mathrm{MnCl}_{2}$ injection via ECA may be the best way to show hind paw stimulation-induced pain in rats, with the best signal-to-noise ratio [3].

Reliable brain activities were observed using the MEMRI method in the control, Torin1-, and XL388-infused groups. Our study presented an integrative analysis of the brain regions, and the $\mathrm{Mn}^{2+}$ enhancements of individual slices were measured and analyzed in the rostral and caudal regions. Integrated signal analysis of the IC showed higher activated signals in the rostral region than in the caudal region of control animals. These results were consistent with previous findings that the rostral part of the IC is more involved in pain processing $[25,26]$. A previous anatomical study [25] revealed that the rostral part of the IC received considerable input from the centrolateral thalamus and mediodorsal thalamus, which showed strong activity in response to noxious stimulus in MEMRI results. The functional characteristics of the caudal part of IC were clearly distinct from those of the rostral part of IC, which has received attention for its role in nociceptive processing [27]. In the rostral part of IC, the majority of activation was observed as it is a site that directly accepts the pain caused by stimulation, while the caudal part of IC may function pathologically with persistent maintenance of mechanical allodynia [28]. In contrast, rats infused with Torin1 or XL388 showed not only a reduction of signals in the rostral part of IC, but also a reduction of signals in the caudal part of IC. In addition to the IC, other cortical target regions, such as the S1HL and $A C C$, have been investigated to evaluate the pain inhibition effects after medication. In the S1HL, which directly reflects noxious stimulation, subcutaneous electrical stimulation in the hind paw could activate $A \beta$ fibers from cutaneous receptors [29]. Evoked pain sensation was reflected in the S1HL. In addition, the ACC was consistently activated in nociception in human and animal studies [30]. Previous research indicated that the neuronal plasticity in ACC is highly correlated with the development of chronic pain [31, 32]. Although the ACC has been associated with different aspects of pain, the precise functional mapping of these aspects still remain largely unknown.

Interestingly, we observed enhanced signals in the M1/2 region, which was not considered to be included in the pain-specific cortical region but was activated by noxious hind paw stimulation. Although the M1/2 is not a part of the pain matrix, it is known that the M1/2 has wide connections to some of the sensory relay nuclei in the thalamus, as well as to efferent and afferent fibers in the spinal cord, that are responsible for the transmission of painful stimuli and modulation of the motor response to noxious contact. Previous investigators have indicated that the motor cortex in rats and mice partially overlaps the $\mathrm{S} 1$ to form a "sensorimotor amalgam" that largely involves representations of the hind limb [33-35]. 
Despite this knowledge, it is difficult to explain how the noxious stimuli in the hind paw activates the $M 1 / 2$. Further research is required to explain the role of the M1/2 in pain perception.

\subsection{How can mTOR inhibition reduce brain activities of chronic pain?}

Modulation of chronic pain through mTOR inhibition is a newly studied application target for chronic pain control $[16,36]$. Previous studies have observed changes in chronic pain through the inhibition of mTOR signaling in the spinal cord [37-40], and recent studies have demonstrated effective modulation of chronic pain by direct mTOR signaling control in the brain $[16,41]$, The activation of mTOR regulates protein synthesis by phosphorylating downstream effectors, which influence a wide range of physiological and pathological states including neuropathic, inflammatory, and cancer-related pain [16-18], The Torin1 and XL388 used in this study were ATP-competitive inhibitors that suppress the phosphorylation of downstream effectors to regulate mRNA translation and protein synthesis [42]. Remarkably, our results for Torin1- and XL388-infused groups showed that the reduced Mn-enhanced signals in the cortical area, such as the IC, S1 HL, M1/2, and ACC. These results demonstrate that mTOR inhibition may have an effect on activation of other brain regions as well as pain reduction through the inhibition of phosphorylation by mTOR control $[13,37]$. Nevertheless, there was no difference in signal enhancement ratios between experimental groups in the $\mathrm{V} 1 / 2$ region. These results indicate that the inhibitory effect of mTOR signaling was not significantly different in response to similar visual cues. Recent studies have suggested that mTOR signaling may be involved in the initiation of pain perception $[15,19]$, which could effectively reduce chronic pain through inhibition of mTOR $[43,44]$. However, the amount of research on how pain is controlled regarding the regulation of mTOR is still insufficient. A better understanding of these signaling pathways would provide great insight into the underlying mechanisms, and lead to more refined therapeutic approaches.

In conclusion, the activity-dependent Mn-enhanced MR signals after mTOR inhibition reported herein indicated that pharmaceutical inhibition of mTOR signaling in the brain could reduce abnormal cortex activities after chronic neuropathic pain. Furthermore, rostral-caudal analysis revealed that the rostral region of IC and M1/2 were more related to pain perceptions. MR imaging studies with manganese ions have many challenges, due to the restrictive pass capability of the blood-brain barrier in addition to difficulties in controlling the accumulation of manganese in tissues and the physiological changes of the body. The development of various studies using improved MR techniques, including MEMRI, have further conveyed diverse and insightful knowledge from fragmentary realizations. These efforts may contribute to future studies of neural mechanisms, and help advance the knowledge of cerebral activation in chronic pain.

\section{Declarations}

\section{Ethics approval and consent to participate}

All animal experiments were approved by the Institutional Animal Care and Use Committee (IACUC) of the Yonsei University Health System (protocol number 2016-0061) 


\section{Consent for publication}

Not applicable

\section{Availability of data and materials}

The datasets used and/or analysed during the current study are available from the corresponding author on reasonable request.

\section{Competing interests}

The authors declare that they have no competing interests

\section{Funding}

This study was supported by the National Research Foundation of Korea (NRF) grant funded by the Korean government (MSIT) (2020R1A2C3008481 and 2019R1I1A1A01059697).

\section{Authors' contributions}

M.C. and B.H.L. designed the study. S.C., K.K., and M.C. conducted research and analyzed data. M.C. and B.H.L. wrote the manuscript. All of the authors read and approved the final manuscript.

\section{Acknowledgements}

We would like to thank Dr. Chan Gyu Joo, Dae Hee Kim, and Hyoung Wook Choi in for their technical assistance with the MRI experiment and data acquisition. We thank the Department of Laboratory Animal Resources, Yonsei Biomedical Research Institute, Yonsei University College of Medicine.

\section{References}

1. Tibbs GR, Posson DJ, Goldstein PA. Voltage-Gated lon Channels in the PNS: Novel Therapies for Neuropathic Pain? Trends in Pharmacological Sciences. 2016;37(7):522-42.

2. Peyron R. Functional brain imaging: what has it brought to our understanding of neuropathic pain? $A$ special focus on allodynic pain mechanisms. Pain. 2016;157 Suppl 1:S67-71.

3. Cha M, Lee K, Lee C, Cho JH, Cheong C, Sohn JH, et al. Manganese-enhanced MR imaging of brain activation evoked by noxious peripheral electrical stimulation. Neurosci Lett. 2016;613:13-8.

4. Devonshire IM, Burston JJ, Xu L, Lillywhite A, Prior MJ, Watson DJG, et al. Manganese-enhanced magnetic resonance imaging depicts brain activity in models of acute and chronic pain: A new window to study experimental spontaneous pain? Neurolmage. 2017;157:500-10.

5. Thompson KJ, Molina RM, Donaghey T, Savaliya S, Schwob JE, Brain JD. Manganese uptake and distribution in the brain after methyl bromide-induced lesions in the olfactory epithelia. Toxicological sciences : an official journal of the Society of Toxicology. 2011;120(1):163-72. 
6. Silva AC, Lee JH, Aoki I, Koretsky APJNiBAIJDttD, Vivo AoMRI. Manganese-enhanced magnetic resonance imaging (MEMRI): methodological and practical considerations. 2004;17(8):532-43.

7. Cha $\mathrm{M}$, Lee $\mathrm{K}$, Won JS, Lee BH. Manganese-enhanced magnetic resonance imaging of the spinal cord in rats with formalin-induced pain. Neuroscience Research. 2019. https://doi.org/https://doi.org/10.1016/j.neures.2019.01.007.

8. Tracey I, Mantyh PWJN. The cerebral signature for pain perception and its modulation. 2007;55(3):377-91.

9. Bingel U, Tracey I. Imaging CNS modulation of pain in humans. Physiology (Bethesda). 2008;23:37180.

10. Jeong $\mathrm{KY}$, Kang JH. Investigation of spinal nerve ligation-mediated functional activation of the rat brain using manganese-enhanced MRI. Exp Anim. 2018;67(1):23-9.

11. Lei B-H, Chen J-H, Yin H-S. Repeated amphetamine treatment alters spinal magnetic resonance signals and pain sensitivity in mice. Neuroscience Letters. 2014;583:70-5.

12. Huang S, Houghton PJ. Targeting mTOR signaling for cancer therapy. Curr Opin Pharmacol. 2003;3(4):371-7.

13. Uttam S, Wong C, Price TJ, Khoutorsky A. elF4E-Dependent Translational Control: A Central Mechanism for Regulation of Pain Plasticity. Front Genet. 2018;9:470.

14. Lyu D, Yu W, Tang N, Wang R, Zhao Z, Xie F, et al. The mTOR signaling pathway regulates pain-related synaptic plasticity in rat entorhinal-hippocampal pathways. Mol Pain. 2013;9:64.

15. Norsted Gregory E, Codeluppi S, Gregory JA, Steinauer J, Svensson Cl. Mammalian target of rapamycin in spinal cord neurons mediates hypersensitivity induced by peripheral inflammation. Neuroscience. 2010;169(3):1392-402.

16. Kwon M, Han J, Kim UJ, Cha M, Um SW, Bai SJ, et al. Inhibition of Mammalian Target of Rapamycin (mTOR) Signaling in the Insular Cortex Alleviates Neuropathic Pain after Peripheral Nerve Injury. Front Mol Neurosci. 2017;10:79.

17. Hay N, Sonenberg NJG, development. Upstream and downstream of mTOR. 2004;18(16):1926-45.

18. Jaworski J, Sheng MJMn. The growing role of mTOR in neuronal development and plasticity. 2006;34(3):205-19.

19. Codeluppi S, Fernandez-Zafra T, Sandor K, Kjell J, Liu Q, Abrams M, et al. Interleukin-6 secretion by astrocytes is dynamically regulated by PI3K-mTOR-calcium signaling. PloS one. 2014;9(3):e92649-e.

20. Cheng NT, Guo A, Cui YP. Intra-articular injection of Torin 1 reduces degeneration of articular cartilage in a rabbit osteoarthritis model. Bone \& joint research. 2016;5(6):218-24.

21. Zhu Y-R, Zhou X-Z, Zhu L-Q, Yao C, Fang J-F, Zhou F, et al. The anti-cancer activity of the mTORC1/2 dual inhibitor XL388 in preclinical osteosarcoma models. Oncotarget. 2016;7(31):49527-38.

22. Cha M, Um SW, Kwon M, Nam TS, Lee BH. Repetitive motor cortex stimulation reinforces the pain modulation circuits of peripheral neuropathic pain. Scientific Reports. 2017;7(1):7986. 
23. Lee BH, Won R, Baik EJ, Lee SH, Moon CH. An animal model of neuropathic pain employing injury to the sciatic nerve branches. Neuroreport. 2000;11(4):657-61.

24. Cloyd RA, Koren SA, Abisambra JF. Manganese-Enhanced Magnetic Resonance Imaging: Overview and Central Nervous System Applications With a Focus on Neurodegeneration. 2018;10(403).

25. Jasmin L, Burkey AR, Granato A, Ohara PT. Rostral agranular insular cortex and pain areas of the central nervous system: a tract-tracing study in the rat. J Comp Neurol. 2004;468(3):425-40.

26. Ohara PT, Granato A, Moallem TM, Wang B-R, Tillet Y, Jasmin LJJoN. Dopaminergic input to GABAergic neurons in the rostral agranular insular cortex of the rat. 2003;32(2):131-41.

27. Jasmin L, Rabkin SD, Granato A, Boudah A, Ohara PT. Analgesia and hyperalgesia from GABAmediated modulation of the cerebral cortex. Nature. 2003;424:316.

28. Benison AM, Chumachenko S, Harrison JA, Maier SF, Falci SP, Watkins LR, et al. Caudal Granular Insular Cortex Is Sufficient and Necessary for the Long-Term Maintenance of Allodynic Behavior in the Rat Attributable to Mononeuropathy. 2011;31(17):6317-28.

29. Lilja J, Endo T, Hofstetter C, Westman E, Young J, Olson L, et al. Blood oxygenation level-dependent visualization of synaptic relay stations of sensory pathways along the neuroaxis in response to graded sensory stimulation of a limb. J Neurosci. 2006;26(23):6330-6.

30. Wager TD, Atlas LY, Lindquist MA, Roy M, Woo CW, Kross E. An fMRI-based neurologic signature of physical pain. N Engl J Med. 2013;368(15):1388-97.

31. Bliss TV, Collingridge GL, Kaang BK, Zhuo M. Synaptic plasticity in the anterior cingulate cortex in acute and chronic pain. Nat Rev Neurosci. 2016;17(8):485-96.

32. Santello M, Nevian T. Dysfunction of cortical dendritic integration in neuropathic pain reversed by serotoninergic neuromodulation. Neuron. 2015;86(1):233-46.

33. Donoghue JP, Kerman KL, Ebner FF. Evidence for two organizational plans within the somatic sensory-motor cortex of the rat. J Comp Neurol. 1979;183(3):647-63.

34. Donoghue JP, Wise SP. The motor cortex of the rat: cytoarchitecture and microstimulation mapping. J Comp Neurol. 1982;212(1):76-88.

35. Young N, Stepniewska I, Kaas J. Chapter 19 - Motor Cortex. In: Watson C, Paxinos G, Puelles L, editors. The Mouse Nervous System. San Diego: Academic Press; 2012. p. 528-38.

36. Lisi L, Aceto P, Navarra P, Dello Russo C. mTOR kinase: a possible pharmacological target in the management of chronic pain. Biomed Res Int. 2015;2015:394257.

37. Zhang W, Sun XF, Bo JH, Zhang J, Liu XJ, Wu LP, et al. Activation of mTOR in the spinal cord is required for pain hypersensitivity induced by chronic constriction injury in mice. Pharmacol Biochem Behav. 2013;111:64-70.

38. Cui J, He W, Yi B, Zhao H, Lu K, Ruan H, et al. mTOR pathway is involved in ADP-evoked astrocyte activation and ATP release in the spinal dorsal horn in a rat neuropathic pain model. Neuroscience. 2014;275:395-403. 
39. Lv J, Li Z, She S, Xu L, Ying Y. Effects of intrathecal injection of rapamycin on pain threshold and spinal cord glial activation in rats with neuropathic pain. Neurol Res. 2015;37(8):739-43.

40. Guo JR, Wang H, Jin XJ, Jia DL, Zhou X, Tao Q. Effect and mechanism of inhibition of $\mathrm{PI3K} / \mathrm{Akt} / \mathrm{mTOR}$ signal pathway on chronic neuropathic pain and spinal microglia in a rat model of chronic constriction injury. Oncotarget. 2017;8(32):52923-34.

41. Um SW, Kim MJ, Leem JW, Bai SJ, Lee BH. Pain-Relieving Effects of mTOR Inhibitor in the Anterior Cingulate Cortex of Neuropathic Rats. Mol Neurobiol. 2018. https://doi.org/10.1007/s12035-0181245-z.

42. Zhuang F, Li M, Gao X, Wang Y, Wang D, Ma X, et al. The antidepressant-like effect of alarin is related to TrkB-mTOR signaling and synaptic plasticity. 2016;313:158-71.

43. Choi S, Kim K, Cha M, Kim M, Lee BH. mTOR signaling intervention by Torin1 and XL388 in the insular cortex alleviates neuropathic pain. Neuroscience Letters. 2020;718:134742.

44. Kim K, Choi S, Cha M, Lee BH. Effects of mTOR inhibitors on neuropathic pain revealed by optical imaging of the insular cortex in rats. Brain Res. 2020;1733:146720.

45. Paxinos G, Watson C. The rat brain in stereotaxic coordinates: hard cover edition: Elsevier; 2006.

\section{Tables}

Table 1. Mean signal intensities of ROIs.

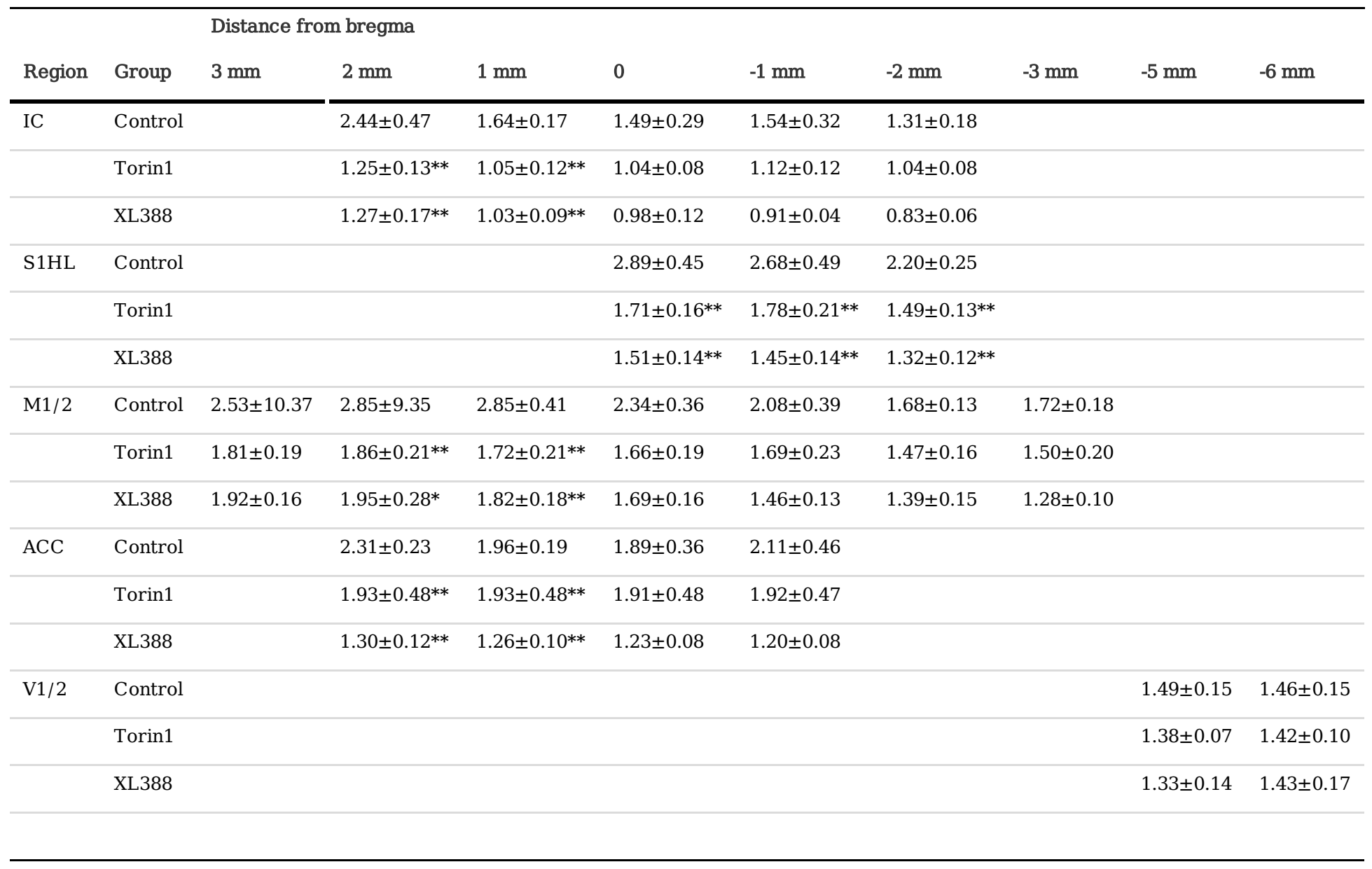




\section{Supplemental Figure Legends}

Supplement Fig. 1. Pain behaviors were assessed before injury and at POD 1, 4, and 7. There was no change in hind paw withdrawal thresholds following sham nerve injury at either before injury and at POD 1, 4, and 7. However, for nerve-injured rats, there was a significant reduction in withdrawal thresholds after nerve injury. Data were analyzed using a paired t-test and two-tailed post-hoc test; error bars represent standard error of the mean; ${ }^{*} P<0.05, * \star \star ~ P<0.0001$.

\section{Figures}

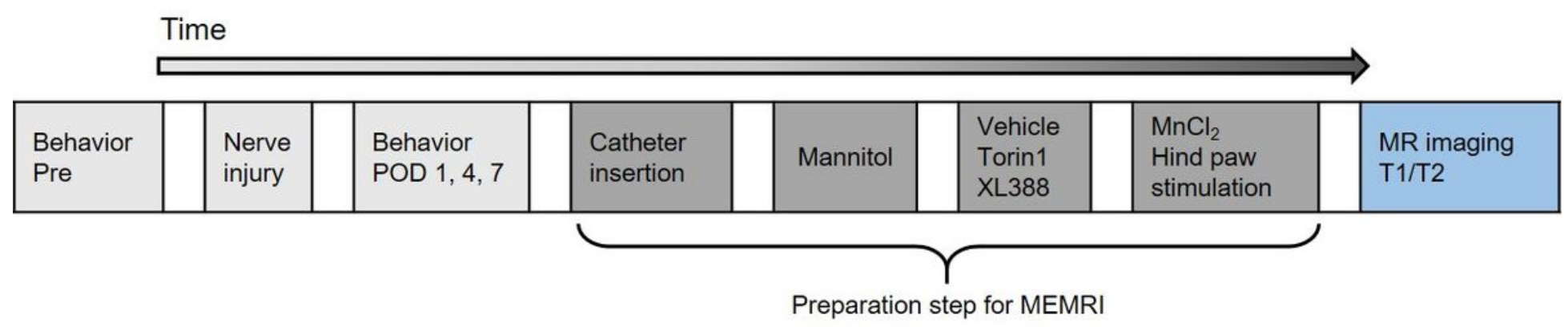

Figure 1

Schematic illustrating the experimental design. All rats underwent behavioral testing prior to nerve injury and at postoperative days (POD) 1, 4, and 7. On each behavioral testing day, the mechanical threshold was assessed using Electronic von Frey. At POD 7, rats were prepared for MEMRI experiments to determine Mn-enhanced changes after evoked pain. MEMRI was performed following T2 scans, and regional $\mathrm{Mn}$ enhancements in the whole brain of experimental groups were compared.

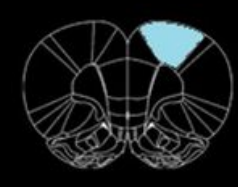

$+3.00 \mathrm{~mm}$

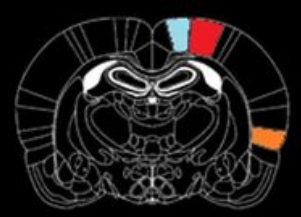

$-2.00 \mathrm{~mm}$

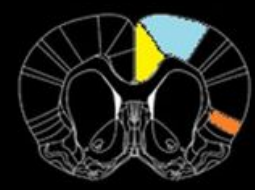

$+2.00 \mathrm{~mm}$

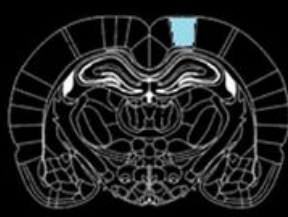

$-3.00 \mathrm{~mm}$

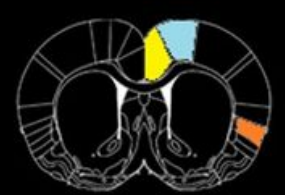

$+1.00 \mathrm{~mm}$

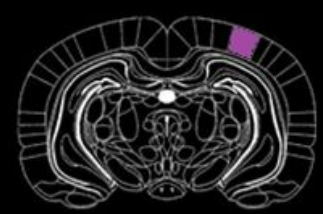

$-5.00 \mathrm{~mm}$

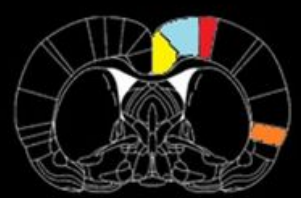

$0.00 \mathrm{~mm}$

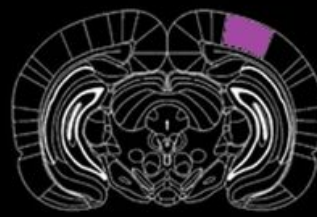

$-6.00 \mathrm{~mm}$

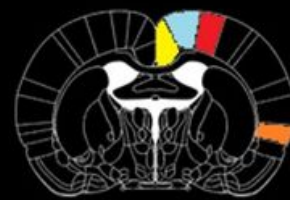

$-1.00 \mathrm{~mm}$

\section{Figure 2}


Representative images and colors of ROls used to calculate average signal intensities. The selected ROIs are shown overlaid on T1 scans in coronal slices (anterior to posterior, respectively). M1/2: motor cortex $1 / 2$, IC: insula, S1HL: primary somatosensory cortex hind limb region, ACC: anterior cingulate cortex, V1/2: visual cortex 1/2. Distances indicated are measurements from bregma according to the Paxinos \& Watson rat brain atlas. [45]
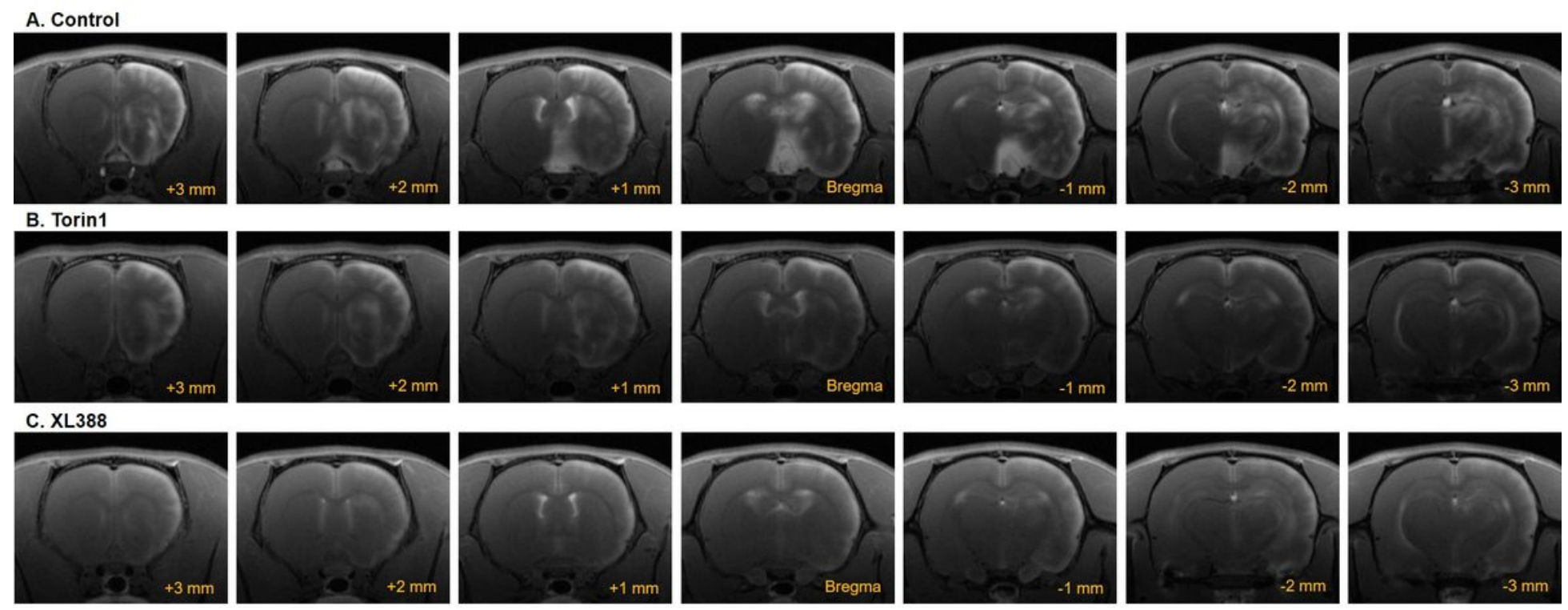

\section{Figure 3}

Comparison of T1-weighted MEMRI following $\mathrm{MnCl} 2$ injection in each group. MR imaging was performed for separate groups: control (vehicle), Torin1-, and XL388-infused with electrical stimulation in the hind paw. In control rats, highly Mn-enhanced signals were observed in the right hemisphere of the brain. However, Torin1- or XL388-infused rats did not show region-specific increased Mn-signals in the brain. Images were obtained from bregam $3 \mathrm{~mm}$ to $-3 \mathrm{~mm}$ MRI scan. 

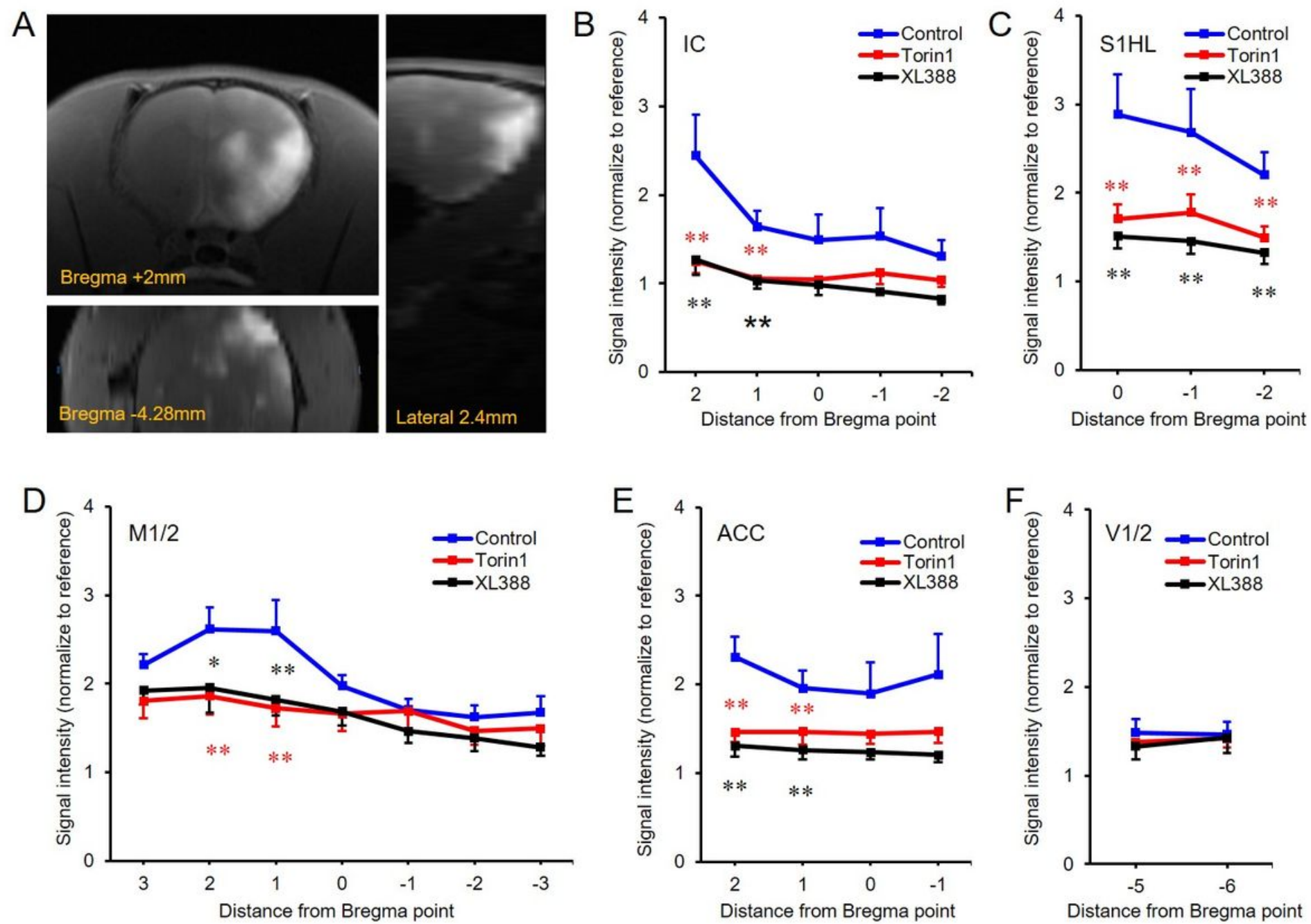

\section{Figure 4}

Mn-enhanced rat brain and regional signal intensity changes. (A) Following administration of $\mathrm{MnCl} 2$, there was a heterogeneous signal distribution, as shown in the coronal (left upper), horizontal (left bottom), and sagittal planes (right) of the rat brain. (B-F) Line graphs depicting the means and standard errors for Mn-enhanced signal intensity in each brain region of the control (blue lines), Torin1 (red lines), and XL388 (black lines) rats. Five regions of Mn-enhanced signal intensity in MR images were analyzed and compared. Signal intensity of Torin1- and XL388-injected rats showed lower intensity compared to control (vehicle injected) rats. ${ }^{*}<<0.05 ;{ }^{*} \mathrm{P}<0.01$. Data are presented as mean $\pm \mathrm{SEM}$. 

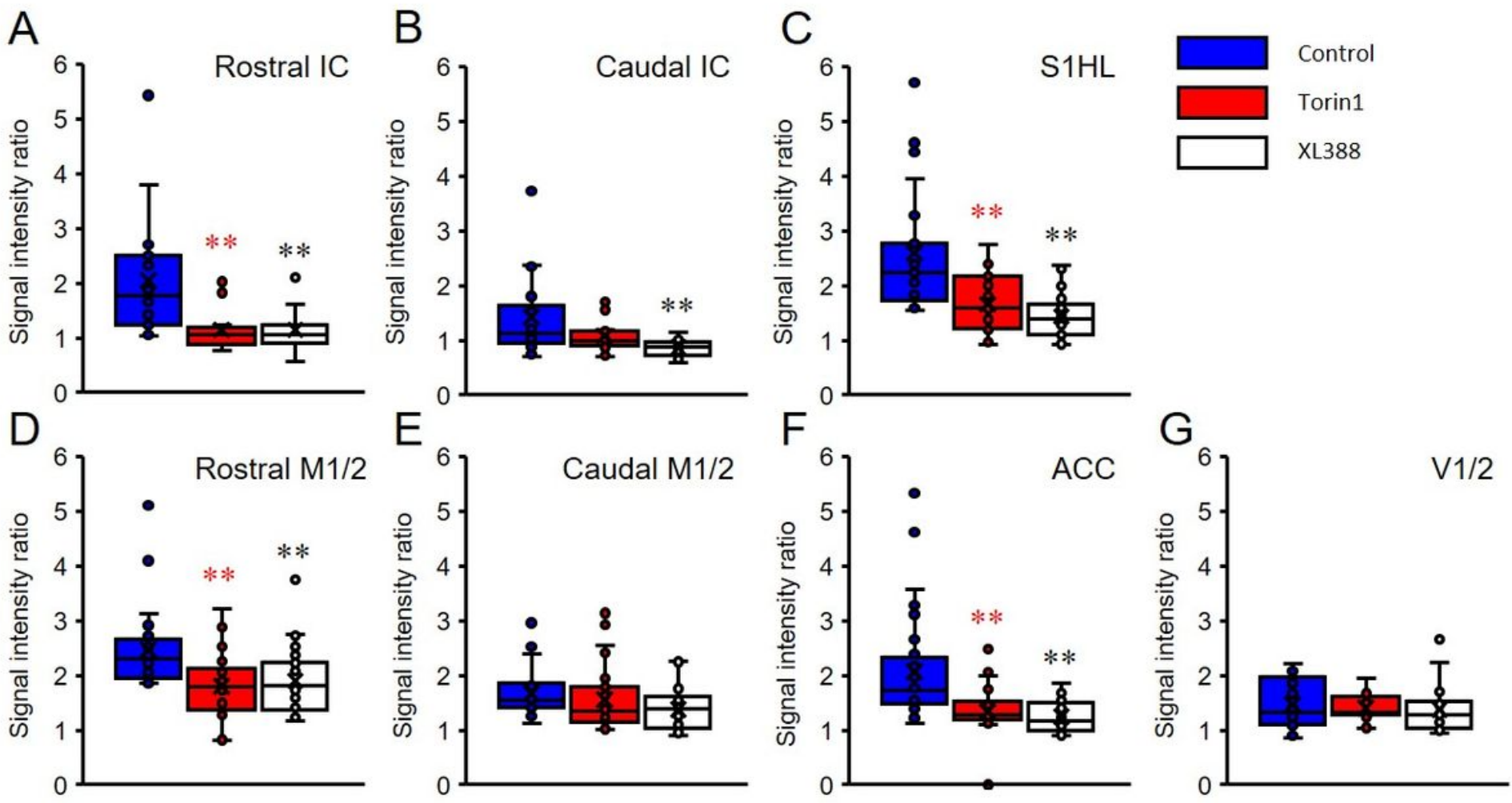

Figure 5

Comparison of $\mathrm{Mn} 2+$ enhancement in the ROls after noxious stimulation. The IC regions were analyzed by rostral-caudal analysis (A and B). S1HL regions showed increased signals in all groups. However, Torin1-, or XL388-treated rats showed reduced signal intensities (C). Torin1- and XL388-treated rats showed significantly reduced signal intensities at the rostral M1/2 area (D and E). In the comparison of ACC, Torin1- and XL388-treated groups showed a significant reduction in signal enhancement (F). However, V1/2 signal did not show any difference within groups (G). Each group is presented by different colors (control: blue, Torin1: red, and XL388: white). ${ }^{*} P<0.05$; ${ }^{\star \star P} P<0.01$. Data are presented as mean \pm SEM.

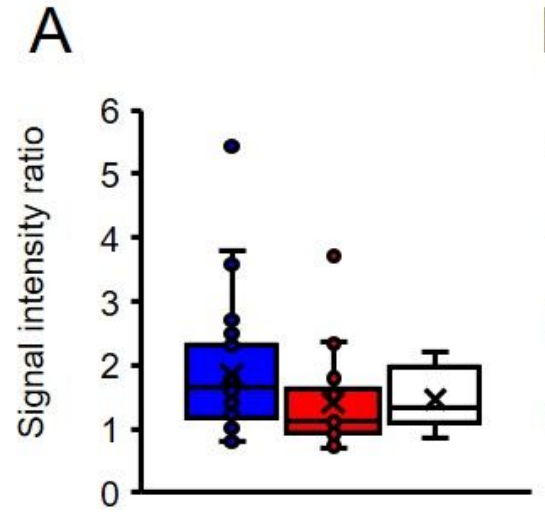

R-IC C-IC V1/2

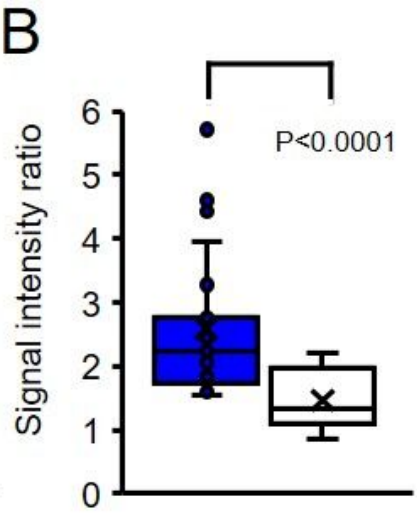

S1HL V1/2

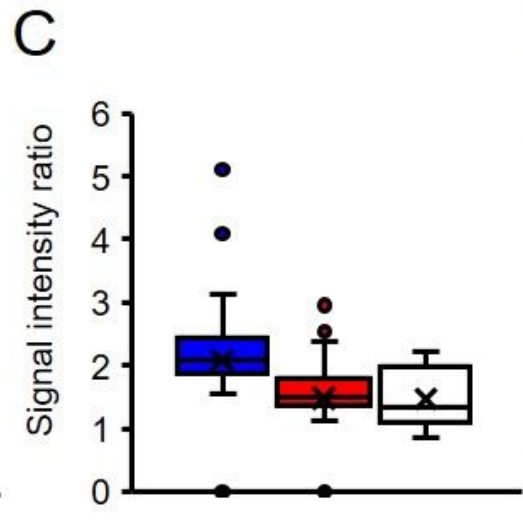

R-M1/2 C-M1/2 V1/2

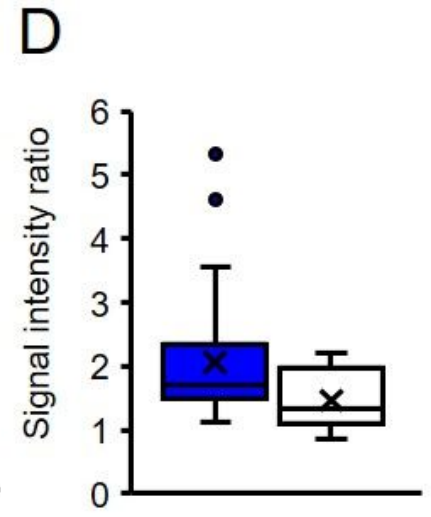

ACC $\quad \mathrm{V} 1 / 2$

Figure 6 
Analysis of stimulation-dependent ROIs in control rats. Relative manganese-enhanced MRI-T1-contrast for the investigated insular (rostral and caudal), S1HL, M1/2 (rostral and caudal), and ACC of brain structures in control (blue or red columns) compared to visual cortex (white columns). Each p-value indicates significant differences between groups. Data are presented as mean \pm SEM.

\section{Supplementary Files}

This is a list of supplementary files associated with this preprint. Click to download.

- S1.jpg 